\title{
RELATIONS BETWEEN SYMMETRIC POWER L-FUNCTIONS AND SPINOR $L$-FUNCTIONS ATTACHED TO IKEDA LIFTS
}

\author{
Kenji Murakawa
}

\begin{abstract}
We prove that the spinor $L$-functions attached to some Siegel cusp forms of degree $\geq 3$, which are Ikeda lifts, have meromorphic continuations and the functional equations.
\end{abstract}

\section{Introduction}

For positive integers $k$ and $n$, let $S_{k}\left(\Gamma_{n}\right)$ be the space of cusp forms with weight $k$ and degree $n, \Gamma_{n}$ denoting the Siegel modular group $\operatorname{Sp}_{n}(\boldsymbol{Z})$ of degree $n$. Around 1996, Duke and Imamog̃lu conjectured the existence of the following lifting which is considered as a generalization of the Saito-Kurokawa lifting (cf. [3]); let $f$ be a normalized Hecke eigenform in the space $S_{2 k}\left(\Gamma_{1}\right)$ and $k \equiv n$ $(\bmod 2)$, then there exists a Hecke eigenform $F$ in $S_{k+n}\left(\Gamma_{2 n}\right)$. The standard $L$ function of $F$ is the product of usual Hecke $L$-functions of $f$ and the Riemann zeta function. In 1999 Ikeda [5] proved that the Duke-Imamog̃lu conjecture was true by using representation theoretic methods and techniques from the theory of Fourier-Jacobi expansions. We call such a Hecke eigenform $F$ the Ikeda lift of $f$. In 2000 Ikeda [6] proved a generalization of the Miyawaki's conjecture [8]: if $g \in S_{k+n+r}\left(\Gamma_{r}\right)$ a Hecke eigenform, then Petterson inner product of $g$ and the Ikeda lift $F$ of $f$ is the Hecke eigenform $\mathscr{F}_{f, g} \in S_{k+n+r}\left(\Gamma_{2 n+r}\right)$. The standard $L$ function of $\mathscr{F}_{f, g}$ is the product of usual Hecke $L$-function of $f$ and the standard $L$-function of $g$. We also call such a Hecke eigenform $\mathscr{F}_{f, g}$ the Ikeda lift of $(f, g)$.

We know that the spinor $L$-function attached to a Hecke eigenform in $S_{k}\left(\Gamma_{n}\right)$ continues meromorphically to all of $\boldsymbol{C}$ and has the functional equation for $n \leq 2$. It is proved for $n=1$ by Hecke, and for $n=2$ by Andrianov [1]. The case of $n \geq 3$ is still unknown.

The purpose of this paper is to give examples of the spinor $L$-function for $n \geq 3$ which has good analytic properties. Such spinor $L$-function is one attached to the Ikeda lift, which is that of even degree $2 n$ expressed as the product of the $m$-th symmetric power $L$-functions for $m \leq n$. Since the $m$-th symmetric power $L$-function for $m \leq 5$ has good analytic properties, the spinor $L$-function

Received April 23, 2001; revised September 26, 2001. 
attached to every Ikeda lift of even degree $\leq 10$ is continued meromorphically to the whole complex $s$-plane. Especially the $m$-th symmetric power $L$-function for $m \leq 3$ has holomorphic continuation, so we have the location of the poles of some spinor $L$-functions in Section 6.

If we assume the meromorphy and the functional equation of the $m$-th symmetric power $L$-functions for all $m \geq 1$, then the spinor $L$-function attached to every Ikeda lift of $f$ has similar analytic properties, and vice versa.

This paper is extracted from author's master thesis in Japanese (Tokyo Institute of Technology, March 2001).

\section{Preliminaries}

Let $f \in S_{2 k}\left(\Gamma_{1}\right)$ be a normalized Hecke eigenform, and for a prime $p$, $\left\{\alpha_{p}, \alpha_{p}^{-1}\right\}$ be the Satake $p$-parameter of $f$ such that

$$
1-a(p) X+p^{2 k-1} X^{2}=\left(1-\alpha_{p} p^{k-1 / 2} X\right)\left(1-\alpha_{p}^{-1} p^{k-1 / 2} X\right),
$$

where $a(p)$ is the $p$-th Fourier coefficient of $f$. Then we define the $m$-th symmetric power $L$-function attached to $f$ by

$$
L_{m}(s, f):=\prod_{i=0}^{m}\left(1-\alpha_{p}^{m-2 i} p^{m(k-1 / 2)-s}\right)^{-1} .
$$

The following conjecture is due to Serre and Langlands;

CONJeCture 2.1 (cf. [10], [13]). Let $f \in S_{2 k}\left(\Gamma_{1}\right)$ be a normalized Hecke eigenform. Put $\tilde{L}_{m}(s, f):=\gamma_{m}(s) L_{m}(s, f)$, where the gamma factor $\gamma_{m}(s)$ is defined by the following relation,

$$
\gamma_{m}(s):= \begin{cases}(2 \pi)^{-r s} \prod_{j=0}^{r-1} \Gamma(s-j(k-1)) & (m=2 r-1) \\ \pi^{-s / 2} \Gamma\left(\frac{s}{2}-\left[\frac{r(k-1)}{2}\right]\right) \gamma_{2 r-1}(s) & (m=2 r) .\end{cases}
$$

Then $\tilde{L}_{m}(s, f)$ can be continued meromorphically to the whole complex s-plane, has the functional equation $\tilde{L}_{m}(s, f)= \pm \tilde{L}_{m}((2 k-1) m+1-s, f)$.

Remark. For an integer $m \leq 5$, it is known that above conjecture is true. Moreover in the case of $m \leq 3$, the function $\tilde{L}_{m}(s, f)$ is entire. It is proved for $m=1$ by Hecke, for $m=2$ by Shimura [12], and for $m=3$ by Shahidi [11].

In general let $F \in S_{k}\left(\Gamma_{n}\right)$ be a Hecke eigenform, and $\left\{\alpha_{p, 0}, \ldots, \alpha_{p, n}\right\}$ be the Satake $p$-parameters of $F$. Then the spinor $L$-function (resp. the standard $L$ function) is defined by $L(s, F, \operatorname{spin})=\prod_{p \text { : prime }} L_{p}(s, F, \operatorname{spin})($ resp. $L(s, F, \mathrm{st})=$ $\left.\prod_{p \text { : prime }} L_{p}(s, F, \mathrm{st})\right)$. Here 


$$
\begin{aligned}
L_{p}(s, F, \text { spin }) & :=\prod_{p}\left[\left(1-\alpha_{p, 0} p^{-s}\right) \prod_{r=1}^{n} \prod_{1 \leq i_{1}<\cdots<i_{r} \leq n}\left(1-\alpha_{p, 0} \alpha_{p, i_{1}} \cdots \alpha_{p, i_{r}} p^{-s}\right)\right]^{-1}, \\
L_{p}(s, F, \mathrm{st}) & :=\prod_{p}\left[\left(1-p^{-s}\right) \prod_{j=1}^{n}\left(1-\alpha_{p, j} p^{-s}\right)\left(1-\alpha_{p, j}^{-1} p^{-s}\right)\right]^{-1} .
\end{aligned}
$$

Now we introduce Ikeda's result.

THeORem $2.2([5])$. Let $n \in N$ with $k \equiv n(\bmod 2)$, let $f \in S_{2 k}\left(\Gamma_{1}\right)$ be a normalized Hecke eigenform. Then there exists a Hecke eigenform $F \in S_{k+n}\left(\Gamma_{2 n}\right)$ such that

$$
L(s, F, \mathrm{st})=\zeta(s) \prod_{j=1}^{2 n} L(s+k+n-j, f) .
$$

Here the Hecke L-function $L(s, f)$ is defined by $\sum_{n \geq 1} a(n) n^{-s}$ with a(n) being the $n$-th Fourier coefficient of $f$.

We call this $F$ the Ikeda lift of $f$.

For an integer $r \geq 1$ with $k \equiv n+r(\bmod 2)$, let $g \in S_{k+n+r}\left(\Gamma_{r}\right)$ be a Hecke eigenform. We put the integral

$$
\mathscr{\mathscr { F }}_{f, g}(Z):=\int_{\Gamma_{r} \backslash \mathscr{H}_{r}} F\left(\left(\begin{array}{cc}
Z & \\
& Z^{\prime}
\end{array}\right)\right) \overline{g\left(Z^{\prime}\right)}\left(\operatorname{det} \operatorname{Im} Z^{\prime}\right)^{k+n-1} d Z^{\prime} \quad\left(Z \in \mathscr{H}_{2 n+r}\right) .
$$

Since the Ikeda lift $F(Z)$ is a cusp form, we have $\mathscr{F}_{f, g} \in S_{k+n+r}\left(\Gamma_{2 n+r}\right)$.

THEOREM $2.3([6])$. Assume that $\mathscr{F}_{f, g}(Z)$ is not identically zero. Then $\mathscr{F}_{f, g}(Z)$ is a Hecke eigenform whose standard L-function is equal to

$$
L\left(s, \mathscr{F}_{f, g}, \mathrm{st}\right)=L(s, g, \mathrm{st}) \prod_{j=1}^{2 n} L(s+k+n-j, f) .
$$

This theorem shows that one of Miyawaki's conjectures [8] is true. We also call this $\mathscr{F}_{f, g}$ the Ikeda lift of $(f, g)$.

\section{Main result}

For $m \in \boldsymbol{Z}_{\geq 0}, n \in \boldsymbol{Z}_{\geq 1}$, and $i_{m, n} \in \boldsymbol{Z}$, the function $r_{m, n}\left(i_{m, n}\right)$ with values in $\boldsymbol{Z}_{\geq 0}$ is defined by

$$
\begin{aligned}
r_{m, n+2}\left(i_{m, n+2}\right) & \\
= & r_{m-2, n}\left(i_{m, n+2}\right)+\left(1+\delta_{m}\right) \cdot r_{m, n}\left(i_{m, n+2}-1\right)+r_{m+2, n}\left(i_{m, n+2}-2\right) \\
& +\sum_{y=-n-2,-n-1, n, n+1}\left\{r_{m-1, n}\left(i_{m, n+2}+y\right)+r_{m+1, n}\left(i_{m, n+2}+y-1\right)\right\} \\
& +\sum_{x=-2 n-1,0,2,2 n+3} r_{m, n}\left(i_{m, n+2}-x\right),
\end{aligned}
$$




$$
\begin{array}{rlll}
r_{0,1}\left(i_{0,1}\right)=1 & \text { if } i_{0,1}=0,1, & r_{0,2}\left(i_{0,2}\right)=1 & \text { if } i_{0,2}=-1,0,1,2,3 \\
r_{1,1}\left(i_{1,1}\right)=1 & \text { if } i_{1,1}=0, & r_{1,2}\left(i_{1,2}\right)=1 & \text { if } i_{1,2}=-1,0,1,2, \\
r_{m, 1}\left(i_{m, 1}\right)=0 & \text { if otherwise, }, & r_{2,2}\left(i_{2,2}\right)=1 & \text { if } i_{2,2}=0, \\
& & r_{m, 2}\left(i_{m, 2}\right)=0 & \text { if otherwise. }
\end{array}
$$

Here $\delta_{m}$ is equal to 0 for $m=0$, and is equal to 1 for $m>0$.

The main result of this paper is the following

Theorem 3.1. Assume $k \equiv n(\bmod 2)$. Let $F_{2 n} \in S_{k+n}\left(\Gamma_{2 n}\right)$ be the Ikeda lift of a normalized Hecke eigenform $f \in S_{2 k}\left(\Gamma_{1}\right)$. Then we have

$$
L\left(s, F_{2 n}, \operatorname{spin}\right)=\prod_{m=0}^{n} \prod_{i_{m, n}} L_{m}\left(s-(n-m) k+i_{m, n}, f\right)^{r_{m, n}\left(i_{m, n}\right)},
$$

where the product is taken over $m(m-1) / 2-n(n-1) / 2 \leq i_{m, n} \leq n(n+1) / 2-$ $m(m+1) / 2$, we regard $L_{0}(s, f)$ as the Riemann zeta function $\zeta(s)$, and $r_{m, n}\left(i_{m, n}\right)$ satisfies the above relations (3.1).

For such a spinor $L$-function $L\left(s, F_{2 n}\right.$, spin), we put

$$
\tilde{L}\left(s, F_{2 n}, \operatorname{spin}\right)=\prod_{m=0}^{n} \prod_{i_{m, n}} \tilde{L}_{m}\left(s-(n-m) k+i_{m, n}, f\right)^{r_{m, n}\left(i_{m, n}\right)} .
$$

Since Conjecture 2.1 is true for $m \leq 5$, by using Theorem 3.1 we obtain

Corollary 3.2. Suppose that $n \leq 5$ with the same notation in Theorem 3.1. Then $\tilde{L}\left(s, F_{2 n}\right.$, spin) has meromorphic continuation to the whole s-plane and satisfies the functional equation

$$
\tilde{L}\left(n k-\frac{n(n+1)}{2}+1-s, F_{2 n}, \text { spin }\right)=(-1)^{k \cdot 2^{n-2}} \tilde{L}\left(s, F_{2 n}, \text { spin }\right) .
$$

Remark. We calculated some poles of $\tilde{L}\left(s, F_{2 n}\right.$, spin) in Section 6 for $n=2,3$.

Conversely suppose the spinor $L$-function attached to $F_{n} \in S_{k}\left(\Gamma_{n}\right)$ of arbitrary even degree $n \in N$ has meromorphic continuation to the whole $s$-plane and the functional equation (3.3), then from Theorem 3.1 it follows that Conjecture 2.1 is true for every cusp form $f$ of weight $2 k$.

\section{Proof of Theorem 3.1}

First, we show the following two lemmas. Let $F_{2 n} \in S_{k+n}\left(\Gamma_{2 n}\right)$ be the Ikeda lift of a normalized Hecke eigenform $f \in S_{2 k}\left(\Gamma_{1}\right)$. 
Lemma 4.1. Let $\left\{\alpha_{p}, \alpha_{p}^{-1}\right\}$ be the Satake p-parameters of $f$, and $\left\{\beta_{p, 0}, \beta_{p, 1}, \ldots, \beta_{p, 2 n}\right\}$ be the Satake p-parameters of $F_{2 n}$. If $k \equiv n(\bmod 2)$, the Satake p-parameters, those are uniquely determined up to the action of the Weyl group, are given for $1 \leq i \leq n$ by

$$
\left\{\begin{aligned}
\beta_{p, 0} & =p^{n k-n(n+1) / 2}, \\
\beta_{p, i} & =\alpha_{p} p^{i-1 / 2} \\
\beta_{p, n+i} & =\alpha_{p}^{-1} p^{i-1 / 2} .
\end{aligned}\right.
$$

Proof. Since $F_{2 n}$ is the Ikeda lift of $f$, the standard $L$-function of $F_{2 n}$ satisfies

$$
L\left(s, F_{2 n}, \mathrm{st}\right)=\zeta(s) \prod_{i=1}^{2 n} L(s+k+n-i, f)
$$

From the definition of the standard $L$-function, for $1 \leq i \leq n$ we obtain the relations

$$
\left\{\begin{aligned}
\beta_{p, i} & =\alpha_{p} p^{i-1 / 2} \\
\beta_{p, n+i} & =\alpha_{p}^{-1} p^{i-1 / 2} .
\end{aligned}\right.
$$

According to [5], there exists the polynomial $\Phi(X) \in C\left[X+X^{-1}\right]$ such that the eigenvalue for Siegel's Eisenstein series $E_{k+n}^{(2 n)}(Z)$ by Hecke operator $T(p)$ is $\Phi\left(p^{k-1 / 2}\right)$. Then the eigenvalue for $F_{2 n}$ by Hecke operator $T(p)$ is equal to $\Phi\left(\alpha_{p}\right)$. The eigenvalue $t(p)$ under the Hecke operator $T(p)$ for some prime $p$ (cf. [4], note the different normalization) is

$$
t(p)=\beta_{p, 0} \sum_{i=0}^{2 n} \phi_{i}\left(\beta_{p, 1}, \ldots, \beta_{p, 2 n}\right),
$$

where $\phi_{i}$ is the $i$-th elementary symmetric polynomial. By substituting the Satake $p$-parameters of Siegel's Eisenstein seris $\left\{1, p^{k+n-1}, p^{k+n-2}, \ldots, p^{k-n}\right\}$ for (4.1), the eigenvalue of $T(p)$ on $E_{k+n}^{(2 n)}(Z)$ is equal to

$$
\begin{aligned}
\prod_{i=1}^{2 n}\left(1+p^{k+n-i}\right) & =\prod_{i=1}^{n}\left(1+p^{k-i}\right)\left(1+p^{k+n-i}\right) \\
& =\left(p^{k-1 / 2}\right)^{n} \prod_{i=1}^{n}\left(p^{k-1 / 2}+p^{-k+1 / 2}+p^{i-i / 2}+p^{-i+1 / 2}\right) .
\end{aligned}
$$

Thus we obtain

$$
\Phi(X)=\left(p^{k-1 / 2}\right)^{n} \prod_{i=1}^{n}\left(X+X^{-1}+p^{i-1 / 2}+p^{-i+1 / 2}\right) .
$$


On the other hand by (4.1) the eigenvalue of $T(p)$ on $F_{2 n}(Z)$ is equal to

$$
\begin{aligned}
t\left(p, F_{2 n}\right) & =\beta_{p, 0} \sum_{i=0}^{2 n} \phi_{i}\left(\alpha_{p} p^{1 / 2}, \ldots, \alpha_{p}^{-1} p^{n-1 / 2}\right) \\
& =\beta_{p, 0} \prod_{i=1}^{n}\left(1+\alpha_{p} p^{i-1 / 2}\right)\left(1+\alpha_{p}^{-1} p^{i-1 / 2}\right) \\
& =\beta_{p, 0} p^{n^{2} / 2}\left(p^{k-1 / 2}\right)^{-n} \Phi\left(\alpha_{p}\right) .
\end{aligned}
$$

Hence it follows from $t\left(p, F_{2 n}\right)=\Phi\left(\alpha_{p}\right)$ that $\beta_{p, 0}=p^{k n-n(n+1) / 2}$.

We have the following lemma by a direct computation.

LEMMA 4.2. For $m \geq 0$

$$
L_{m}^{(p)}\left(\alpha_{p} X, f\right) L_{m}^{(p)}\left(\alpha_{p}^{-1} X, f\right)=L_{m+1}^{(p)}\left(p^{-k+1 / 2} X, f\right) L_{m-1}^{(p)}\left(p^{k-1 / 2} X, f\right) .
$$

For $m \geq 1$

$$
L_{m}^{(p)}\left(\alpha^{2} X, f\right) L_{m}^{(p)}\left(\alpha^{-2} X, f\right)=L_{m+2}^{(p)}\left(p^{-2 k+1} X, f\right) L_{m-2}^{(p)}\left(p^{2 k-1} X, f\right) .
$$

where $L_{m}^{(p)}(X, f)=1(m=-1)$.

Proof of Theorem 3.1. We prove the main theorem by induction on the degree $n$. From the assumption, we put $k \equiv n(\bmod 2)$. For $n=1$ an integer $k$ is odd, and the Ikeda lift $F_{2}$ satisfies (3.2) by the Saito-Kurokawa lifting, whose spinor $L$-function is

$$
L\left(s, F_{2}, \operatorname{spin}\right)=L(s, f) \zeta(s-k) \zeta(s-k+1) .
$$

When $n=2$, the integer $k$ is even, by using the definition of the spinor $L$-function and Lemma 4.1, the spinor $L$-function of the Ikeda lift $F_{4}$ is

$$
L\left(s, F_{4}, \operatorname{spin}\right)=L_{2}(s, f) \prod_{-1 \leq i \leq 2} L(s-k+i, f) \prod_{-1 \leq j \leq 3} \zeta(s-2 k+j) .
$$

Let $F_{2 n} \in S_{k+n}\left(\Gamma_{2 n}\right)$, and $F_{2(n+2)} \in S_{k+n+2}\left(\Gamma_{2(n+2)}\right)$ be the Ikeda lifts of a normalized Hecke eigenform $f \in S_{2 k}\left(\Gamma_{1}\right)$. Suppose the Ikeda lift $F_{2 n}$ satisfies (3.2), then by Lemma 4.1 the Satake $p$-parameters of $F_{2 n}$ are given by

$$
\begin{gathered}
\beta_{p, 0}=p^{n k-n(n+1) / 2}, \\
\left\{\beta_{p, 1}, \ldots, \beta_{p, 2 n}\right\}=\left\{\alpha_{p} p^{1-1 / 2}, \ldots, \alpha_{p} p^{n-1 / 2}, \alpha_{p}^{-1} p^{1-1 / 2}, \ldots, \alpha_{p}^{-1} p^{n-1 / 2}\right\},
\end{gathered}
$$

and the Satake $p$-parameters of $F_{2(n+2)}$ are given by

$$
\gamma_{p, 0}=p^{n k-n(n+1) / 2} p^{2 k-2 n-3},
$$




$$
\begin{aligned}
& \left\{\gamma_{p, 1}, \ldots, \gamma_{p, 2(n+2)}\right\} \\
& \quad=\left\{\alpha_{p} p^{1-1 / 2}, \ldots, \alpha_{p}^{-1} p^{n-1 / 2}, \alpha_{p} p^{n+1 / 2}, \alpha_{p} p^{n+3 / 2}, \alpha_{p}^{-1} p^{n+1 / 2}, \alpha_{p}^{-1} p^{n+3 / 2}\right\} .
\end{aligned}
$$

From that, we obtain the spinor $L$-function of $F_{2(n+2)}$ :

$$
\begin{aligned}
L_{p}(X, & \left.F_{2(n+2)}, \text { spin }\right) \\
= & \prod_{x=-2 n-1,0,1,2,2 n+3} L_{p}\left(p^{2 k-x} X, F_{2 n}, \text { spin }\right) \\
& \times \prod_{y=-n-2,-n-1, n, n+1} L_{p}\left(p^{2 k+y-1 / 2} \alpha_{p} X, F_{2 n}, \text { spin }\right) L_{p}\left(p^{2 k+y-1 / 2} \alpha_{p}^{-1} X, F_{2 n}, \text { spin }\right) \\
& \times L_{p}\left(\alpha_{p}^{2} p^{2 k-1} X, F_{2 n}, \operatorname{spin}\right) L_{p}\left(p^{2 k-1} X, F_{2 n}, \text { spin }\right) L_{p}\left(\alpha_{p}^{-2} p^{2 k-1} X, F_{2 n}, \text { spin }\right) .
\end{aligned}
$$

The assumption and Lemma 4.2 lead that

$$
\begin{aligned}
L\left(s, F_{2(n+2)}, \text { spin }\right) & \prod_{x=-2 n-1,0,1,2,2 n+3} \prod_{m=0}^{n} \prod_{i_{m, n}} L_{m}\left(s-(n+2-m) k+i_{m, n}+x, f\right)^{r_{m, n}\left(i_{m, n}\right)} \\
& \times \prod_{y=-n-2,-n-1, n, n+1} \prod_{m=1}^{n+1} \prod_{i_{m-1, n}} L_{m}\left(s-(n+2-m) k+i_{m-1, n}-y, f\right)^{r_{m-1, n}\left(i_{m-1, n}\right)} \\
& \times \prod_{y=-n-2,-n-1, n, n+1} \prod_{m=0}^{n-1} \prod_{i_{m+1, n}} L_{m}\left(s-(n+2-m) k+i_{m+1, n}-y+1, f\right)^{r_{m+1, n}\left(i_{m+1, n}\right)} \\
& \times \prod_{m=2}^{n+2} \prod_{i_{m-2, n}} L_{m}\left(s-(n+2-m) k+i_{m-2, n}, f\right)^{r_{m-2, n}\left(i_{m-2, n}\right)} \\
& \times \prod_{m=1}^{n} \prod_{i_{m, n}} L_{m}\left(s-(n+2-m) k+i_{m, n}+1, f\right)^{r_{m, n}\left(i_{m, n}\right)} \\
& \times \prod_{m=0}^{n-2} \prod_{i_{m+2, n}} L_{m}\left(s-(n+2-m) k+i_{m+2, n}+2, f\right)^{r_{m+2, n}\left(i_{m+2, n}\right)} \\
= & \prod_{m=0}^{n+2} \prod_{i_{m, n+2}} L_{m}\left(s-(n+2-m) k+i_{m, n+2}, f\right)^{r_{m, n+2}\left(i_{m, n+2}\right)} .
\end{aligned}
$$

Here the sum is taken over interval $m(m-1) / 2-(n+2)(n+1) / 2 \leq i_{m, n+2} \leq$ $(n+2)(n+3) / 2-m(m+1) / 2$, and $r_{m, n+2}\left(i_{m, n+2}\right)$ satisfies (3.1). This proves Theorem 3.1. 


\section{The Ikeda lift of odd degree}

For $m \in \boldsymbol{Z}_{\geq 0}, n \in \boldsymbol{Z}_{\geq 1}$, and $i_{m, n} \in \boldsymbol{Z}$, the function $t_{m, n}\left(i_{m, n}\right)$ with values in $\boldsymbol{Z}_{\geq 0}$ is defined by

$$
\begin{aligned}
t_{m, n+2}\left(i_{m, n+2}\right)= & \sum_{x=-2 n-1,0,2,2 n+3} t_{m, n}\left(i_{m, n+2}-x\right) \\
& +\sum_{y=-n-2,-n-1, n, n+1} t_{m-1, n}\left(i_{m, n+2}+y\right) \\
& +t_{m-2, n}\left(i_{m, n+2}\right),
\end{aligned}
$$

For Siegel's Eisenstein series $E_{k+n+r}^{(2 n+2 r)}$, the integral

$$
\int_{\Gamma_{r} \backslash \mathscr{H}_{r}} E_{k+n+r}^{(2 n+2 r)}\left(\left(\begin{array}{cc}
Z & \\
& Z^{\prime}
\end{array}\right)\right) \overline{g\left(Z^{\prime}\right)}\left(\operatorname{det} \operatorname{Im} Z^{\prime}\right)^{k+n-1} d Z^{\prime}
$$

is a scalar multiple of the Klingen's Eisenstein series $[g]_{r}^{2 n+r}(Z)$ (cf. [2]).

If $r$ is odd in Theorem 2.3, the Ikeda lift $\mathscr{F}_{f, g}(Z)$ of $(f, g)$ is a Hecke eigenform with odd degree $2 n+r$ and weight $k+n+r$. Let $r=1$, and we argue in the same way as the proof of Theorem 3.1 and replacing Siegel's Eisenstein series by Klingen's Eisenstein series. Then we have the following theorem.

THEOREM 5.1. Assume $k \equiv n+1(\bmod 2)$. Let $f \in S_{2 k}\left(\Gamma_{1}\right), g \in S_{k+n+1}\left(\Gamma_{1}\right)$ be normalized Hecke eigenforms, and $F_{2 n+1} \in S_{k+n+1}\left(\Gamma_{2 n+1}\right)$ be the Ikeda lift of $(f, g)$. Then we have

$$
L\left(s, F_{2 n+1}, \text { spin }\right)=\prod_{m=0}^{n} \prod_{i_{m, n}} L(s-(n-m) k+i_{m, n}, g \times \underbrace{f \times \cdots \times f}_{m \text { times }})^{t_{m, n}\left(i_{m, n}\right)},
$$

where the product is taken over $m(m-1) / 2-n(n-1) / 2 \leq i_{m, n} \leq n(n+1) / 2-$ $m(m+1) / 2$, and $t_{m, n}\left(i_{m, n}\right)$ satisfies the above relations $(5.1)$.

\section{Examples}

We calculate $r_{m, n}\left(i_{m, n}\right)$ for the spinor $L$-function appearing in Theorem 3.1, and compare with the following Andrianov's conjecture about the spinor $L$ function. 
Conjecture 6.1 (cf. [1]). Let $F \in S_{k}\left(\Gamma_{n}\right)$ be a Hecke eigenform. We set

$$
\hat{L}(s, F, \text { spin }):=(2 \pi)^{-2^{n-1} s} \gamma_{n, k}(s) L(s, F, \text { spin }),
$$

where the $\gamma_{n, k}(s)$ are defined by the relations

$$
\gamma_{1, k}(s)=\Gamma(s), \quad \gamma_{n, k}(s)=\gamma_{n-1, k}(s-k+n) \gamma_{n-1, k}(s) \quad(n>1) .
$$

Then $\hat{L}(s, F, \operatorname{spin})$ can be meromorphically continued to the whole complex s-plane, and the following functional equation holds

$$
\hat{L}\left(n k-\frac{n(n+1)}{2}+1-s, F, \text { spin }\right)=(-1)^{k \cdot 2^{n-2}} \hat{L}(s, F, \text { spin }) .
$$

By calculating $r_{m, n}\left(i_{m, n}\right)$, we have the following analytic properties of the spinor $L$-function attached to the Ikeda lift with degree 3, 4, 5, 6 and 8 .

The case of degree 4. Let $k$ be even, and $F_{4} \in S_{k+2}\left(\Gamma_{4}\right)$ be the Ikeda lift of $f \in S_{2 k}\left(\Gamma_{1}\right)$. We have the spinor $L$-function of $F_{4}$ by (4.2) in the previous section. The function $\tilde{L}\left(s, F_{4}\right.$, spin) is meromorphically continued to the whole complex $s$-plane, and has at most simple poles at the points $s=2 k+2,2 k+1$, $2 k-1,2 k-2$, and satisfies the functional equation

$$
\tilde{L}\left(s, F_{4}, \operatorname{spin}\right)=\tilde{L}\left(4 k-1-s, F_{4}, \text { spin }\right) .
$$

The case of degree 6. Let $k$ be odd, and $F_{6} \in S_{k+3}\left(\Gamma_{6}\right)$ be the Ikeda lift of $f \in S_{2 k}\left(\Gamma_{1}\right)$. Then the spinor $L$-function of $F_{6}$ satisfies

$$
\begin{aligned}
L\left(s, F_{6}, \text { spin }\right)= & L_{3}(s, f) \prod_{-2 \leq i_{2,3} \leq 3} L_{2}\left(s-k+i_{2,3}, f\right) \\
& \times \prod_{-3 \leq i_{1,3} \leq 5} L\left(s-2 k+i_{1,3}, f\right)^{r_{1,3}\left(i_{1,3}\right)} \prod_{-3 \leq i_{0,3} \leq 6} \zeta\left(s-3 k+i_{0,3}\right)^{r_{0,3}\left(i_{0,3}\right)},
\end{aligned}
$$

where the exponent $r_{m, 3}\left(i_{m, 3}\right)$ are the following value;

$$
r_{1,3}\left(i_{1,3}\right)=\left\{\begin{array}{ll}
1 & i_{1,3}=-3,-2,4,5 \\
2 & i_{1,3}=-1,0,1,2,3
\end{array}, \quad r_{0,3}\left(i_{0,3}\right)=\left\{\begin{array}{ll}
1 & i_{0,3}=-3,-2,-1,4,5,6 \\
2 & i_{0,3}=0,1,2,3
\end{array} .\right.\right.
$$

The function $\tilde{L}\left(s, F_{6}\right.$, spin $)$ is meromorphically continued to the whole complex $s$ plane, and has at most simple poles at the point $s=3 k+4$. The functional equation of $\tilde{L}\left(s, F_{6}\right.$, spin $)$ is equal to

$$
\tilde{L}\left(s, F_{6}, \text { spin }\right)=\tilde{L}\left(6 k-2-s, F_{6}, \text { spin }\right) .
$$

The case of degree 8. Let $k$ be even, and $F_{8} \in S_{k+4}\left(\Gamma_{8}\right)$ be the Ikeda lift of $f \in S_{2 k}\left(\Gamma_{1}\right)$. Then the spinor $L$-function of $F_{8}$ satisfies

$$
\begin{aligned}
L\left(s, F_{8}, \text { spin }\right)= & L_{4}(s, f) \prod_{-3 \leq i_{3,4} \leq 4} L_{3}\left(s-k+i_{3,4}, f\right) \prod_{-5 \leq i_{2,4} \leq 7} L_{2}\left(s-2 k+i_{2,4}\right)^{r_{2,4}\left(i_{2,4}\right)} \\
& \times \prod_{-6 \leq i_{1,4} \leq 9} L\left(s-3 k+i_{1,4}\right)^{r_{1,4}\left(i_{1,4}\right)} \prod_{-6 \leq i_{0,4} \leq 10} \zeta\left(s-4 k+i_{0,4}\right)^{r_{0,4}\left(i_{0,4}\right)},
\end{aligned}
$$


where the exponent $r_{m, 4}\left(i_{m, 4}\right)$ are the following value;

$$
\begin{gathered}
r_{2,4}\left(i_{2,4}\right)=\left\{\begin{array}{ll}
1 & i_{2,4}=-5,-4,6,7 \\
2 & i_{2,4}=-3,-2,4,5 \\
3 & i_{2,4}=-1,0,1,2,3
\end{array}, \quad r_{1,4}\left(i_{1,4}\right)= \begin{cases}1 & i_{1,4}=-6,-5,8,9 \\
2 & i_{1,4}=-4,7 \\
3 & i_{1,4}=-3,-2,5,6 \\
4 & i_{1,4}=-1,4 \\
5 & i_{1,4}=0,1,2,3\end{cases} \right. \\
r_{0,4}\left(i_{0,4}\right)= \begin{cases}1 & i_{0,4}=-6,-5,-4,8,9,10 \\
2 & i_{0,4}=-3,7 \\
3 & i_{0,4}=-2,-1,5,6 \\
4 & i_{0,4}=0,1,2,3,4\end{cases}
\end{gathered}
$$

The function $\tilde{L}\left(s, F_{8}\right.$, spin) is meromorphically continued to the whole complex $s$-plane and has the functional equation

$$
\tilde{L}\left(s, F_{8}, \text { spin }\right)=\tilde{L}\left(8 k-3-s, F_{8}, \text { spin }\right) .
$$

The case of degree 3. Let $k$ be even, and $F_{3} \in S_{k+2}\left(\Gamma_{3}\right)$ be the Ikeda lift of $(f, g) \in S_{2 k}\left(\Gamma_{1}\right) \times S_{k+2}\left(\Gamma_{1}\right)$. Then the spinor $L$-function of $F_{3}$ satisfies

$$
L\left(s, F_{3}, \operatorname{spin}\right)=L(s, f \times g) L(s-k, g) L(s-k+1, g) .
$$

The function $\tilde{L}\left(s, F_{3}\right.$, spin) is holomorphically continued to the whole complex $s$ plane, and satisfies the functional equation

$$
\tilde{L}\left(s, F_{3}, \operatorname{spin}\right)=\tilde{L}\left(3 k+1-s, F_{3}, \text { spin }\right) .
$$

The case of degree 5. Let $k$ be odd, and $F_{5} \in S_{k+3}\left(\Gamma_{5}\right)$ be the Ikeda lift of $(f, g) \in S_{2 k}\left(\Gamma_{1}\right) \times S_{k+3}\left(\Gamma_{1}\right)$. Then the spinor $L$-function of $F_{5}$ satisfies

$$
\begin{aligned}
& L\left(s, F_{5}, \text { spin }\right) \\
& \quad=L(s, f \times f \times g) \prod_{-1 \leq i_{1,2} \leq 2} L\left(s-k+i_{1,2}, f \times g\right) \prod_{i_{0,2}=-1,0,2,3} L\left(s-2 k+i_{0,2}, g\right) .
\end{aligned}
$$

The function $\tilde{L}\left(s, F_{5}, \operatorname{spin}\right)$ is holomorphically continued to the whole complex $s$ plane, and satisfies the functional equation

$$
\tilde{L}\left(s, F_{5}, \operatorname{spin}\right)=\tilde{L}\left(5 k+1-s, F_{5}, \operatorname{spin}\right) .
$$

For $n=3,4,5,6,8$, we put

$$
R_{n}(s):=\frac{\tilde{L}\left(s, F_{n}, \operatorname{spin}\right)}{\hat{L}\left(s, F_{n}, \operatorname{spin}\right)},
$$

then $R_{n}(s)$ is the ratio of gamma function, and also satisfies the functional equation $R_{n}(s)=R_{n}(n k-n(n+1) / 2+1-s)$. This shows that our theorem satisfies Andrianov's conjecture [1, p. 115] on the spinor $L$-function. 
Acknowledgments. I am thankful to Professor S. Mizumoto for his advice, encouragement and patient support in all the process. Also I am thankful to Professor T. Ikeda for his valuable comments.

I am grateful to my family and all of my friends for their constant encouragement.

\section{REFERENCES}

[1] A. N. Andrianov, Euler products corresponding to Siegel modular forms of genus 2, Russian Math. Surveys, 29 (1974), (3), 45-116.

[2] S. BÖCHERER, Über die Funktionalgleichung automorpher $L$-Funktionen zur Siegelschen Modulgruppe, J. Reine Angew. Math., 362 (1985), 146-168.

[ 3 ] S. Breulmann and M. Kuss, On a conjecture of Duke-Imamoḡlu, Proc. Amer. Math. Soc., 128 (2000), 1595-1604.

[4] E. Freitag, Siegelsche Modulfunktionen, Grundlehren Math. Wiss. 254, Springer-Verlag, Berlin, 1983.

[5] T. IKEDA, On the lifting of elliptic cusp forms to Siegel cusp forms of degree $2 n$, preprint, 1999.

[6] T. IKeDA, On some construction of Siegel cusp forms: Miyawaki's conjecture, preprint, 2000.

[ 7] W. KoHnen, Lifting modular forms of half-integral weight to Siegel modular forms of even genus, preprint, 2000.

[ 8 ] I. Miyawaki, Numerical examples of Siegel cusp forms of degree 3 and their zeta-functions, Mem. Fac. Sci. Kyushu Univ. Ser. A, 46 (1992), 307-339.

[9] R. A. Rankin, Contributions to the theory of Ramanujan's function $\tau(n)$ and similar arithmetical functions, Proc. Cambridge Philos. Soc., 35 (1939), (I) 351-356, (II) 357-372.

[10] J.-P. SERre, Une interprétation des congruences relatives à la fonction $\tau$ de Ramanujan, Séminaire Delange-Pisot-Poitou: 1967/68, Théorie des Nombres, Secrétariat mathématique, Paris, 1969.

[11] F. Shahidi, Third symmetric power $L$-functions for $G L(2)$, Compositio Math., 70 (1989), $245-273$.

[12] G. Shimura, On the holomorphy of certain Dirichlet series, Proc. London Math. Soc. (3), 31 (1975), 79-98.

[13] D. ZAGIER, Modular forms whose Fourier coefficients involve zeta-functions of quadratic fields, Modular Functions of One Variable VI, Bonn 1976 (J.-P. Serre and D. B. Zagier eds.), Lecture Notes in Math. 627, Springer-Verlag, Berlin, 1977, 105-169.

The Taiyo Mutual Life Insurance Co.

7-7 Nibancho, Chiyoda-KU,

TOKYO 102-0084, JAPAN 